\title{
Compromiso neurológico y cardiovascular en pacientes con triquinosis
}

\author{
Recibido: 29/5/19 Aceptado: 9/7/19
}

Lucía Lamponi Tappatá, María Laura Spadaro, Diego Maurizi, Adriana Montanini.

\section{RESUMEN}

Introducción: La triquinosis es una infección parasitaria causada por nematodos del género Trichinella. El compromiso cardíaco no es habitual, pero representa la causa más frecuente de muerte por triquinosis, mientras que la afectación neurológica ocurre principalmente en pacientes severamente enfermos. Se sabe que a mayor cantidad de larvas ingeridas menor es el tiempo de incubación y mayor es la severidad de la enfermedad. Se presentan dos pacientes con compromiso del sistema nervioso central, uno de ellos cardiovascular, ambos pertenecientes a un brote de triquinosis ocurrido en la ciudad de Bahía Blanca.

Palabras clave: triquinosis, Trichinella spiralis, infección por nematodos.
Unidad de Infectología, Hospital Municipal de Agudos Dr. Leónidas Lucero. Bahía Blanca, Argentina.

Dirección para correspondencia:

Lucía Lamponi Tappatá. Florida 1323, dep D. CP 8000. Bahía Blanca. Provincia de Buenos Aires. lucialamponit@gmail.com

El protocolo de trabajo fue aprobado por un comité de ética y de investigación y se obtuvo consentimiento informado por escrito para cada uno de los pacientes incluidos.

Los autores declaran que no existieron fuentes de financiamiento ni conflictos de interés entorno a esta publicación. 


\section{Introducción}

La triquinosis es una infección parasitaria causada por nematodos del género Trichinella. Los cerdos son la fuente de infección más frecuente en humanos y la ingesta de carne mal cocida constituye la principal forma de transmisión (1). El compromiso cardíaco no es habitual, pero representa la causa más frecuente de muerte por triquinosis. Si bien las larvas no se enquistan en el músculo cardíaco, sí lo invaden y generan respuesta inflamatoria y miocarditis $(2$, 3). Las manifestaciones neurológicas pueden desarrollarse tempranamente o de forma tardía y pueden ser de naturaleza difusa o focal. La tomografía y la resonancia magnética pueden mostrar lesiones pequeñas multifocales localizadas en la corteza cerebral y sustancia blanca (4).

Se sabe que a mayor cantidad de larvas ingeridas menor es el tiempo de incubación y mayor la severidad de la enfermedad. La infección leve (menos de 10 larvas/gramo de músculo) suele ser asintomática, mientras que la severa puede incluir compromiso cardíaco, del sistema nervioso central, renal y/o pulmonar (5).

Se presentan dos pacientes con triquinosis con compromiso del sistema nervioso central, uno de ellos además con manifestaciones cardíacas, que formaron parte de un brote ocurrido en la ciudad de Bahía Blanca durante julio y agosto de 2018. El mismo fue a partir del consumo de chacinados sin rótulo y afectó a 38 personas cuyo diagnóstico fue realizado por epidemiología, cuadro clínico compatible y serología confirmatoria. Los chacinados responsables del brote resultaron positivos para Trichinella spiralis en el análisis por digestión enzimática artificial, con una carga parasitaria de 550 larvas/gramo.

\section{Caso 1. Paciente con compromiso del sistema nervioso central y cardiovascular}

Paciente masculino de 45 años, tabaquista, enolista y obeso. Fue llevado a la guardia en julio de 2018 por imposibilidad para comunicarse y debilidad en hemicuerpo derecho de tres horas de evolución. Presentaba además cuadro de disnea y dolor precordial al momento de la consulta. Al interrogatorio dirigido refirió diarrea, vómitos y debilidad las últimas 72 horas. La familia del paciente relató que había consumido chacinados caseros 24 horas previas al inicio de la diarrea. Al examen físico se constató Glasgow 13/15, afasia de expresión y paresia braquio crural derecha grado I, con piel y mucosas hipohidratadas. Se realizó electrocardiograma que mostró supradesnivel del ST en cara anterior extensa. Se realizó laboratorio que informó hiponatremia (sodio 124 $\mathrm{mmol} / \mathrm{L}$ ) e insuficiencia renal (urea $108 \mathrm{mg} / \mathrm{dL}$, creatinina $7,27 \mathrm{mg} / \mathrm{dL}$ ). Hemograma sin eosinofilia. Enzimas cardíacas: creatinfosfoquinasa $1414 \mathrm{U} / \mathrm{l}$, fracción MB $66 \mathrm{U} / \mathrm{l}$, troponina T 8096 ng/l. Se realizó tomografía de encéfalo que mostró hipodensidad córtico-subcortical en lóbulo frontal derecho y a nivel parieto-occipital izquierdo (ver Figura 1).

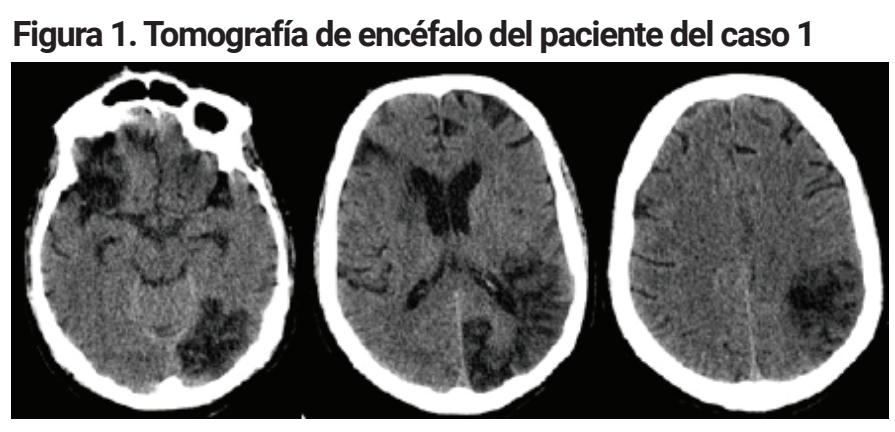

Se observa hipodensidad córtico-subcortical de tipo isquémica en lóbulo frontal derecho y a nivel parieto-occipital izquierdo. Del lado derecho compromete la sustancia blanca profunda y núcleos grises, afectando la cabeza del núcleo caudado, el brazo anterior de la cápsula interna y el putamen ipsilateral.

Se interpretó el cuadro como infarto agudo de miocardio y accidente cerebrovascular isquémico y pasó a unidad de terapia intensiva. Evolucionó con deterioro del sensorio, requiriendo ventilación mecánica invasiva. Por no tener disponibilidad inmediata para realizar cinecoronariografía se administró terapia fibrinolítica, constatando mejoría de los cambios electrocardiográficos. Por sospecha de triquinosis recibió tratamiento específico con mebendazol $400 \mathrm{mg} / 8$ hs por diez días asociado a corticoides. Se interpretó la hiponatremia y la insuficiencia renal severa producto de la deshidratación. Se realizó serología IgG para Triquinella spiralis, resultando negativa al inicio y positiva a las dos semanas (título 1/64).

El paciente se trasladó a clínica médica con miopatía severa del paciente crítico y con secuela neurológica focal. Recibió el alta médica luego de 69 días de internación. Actualmente el paciente persiste con la secuela neurológica focal y con debilidad generalizada. 


\section{Caso 2. Paciente con compromiso del sistema nervioso central}

Paciente de sexo masculino de 57 años, enolista, que consultó en julio de 2018 a la guardia por diarrea, mialgias y deterioro del estado general. Al interrogatorio dirigido refirió consumo de chacinados caseros ocho días previos al inicio de los síntomas. Al examen físico se constató desorientación temporo-espacialy pielymucosas hipohidratadas. Evolucionó con episodios de incontinencia urinaria. Se realizó laboratorio que informó aumento de creatinfosfoquinasa (309 U/I) y eosinofilia $\left(23 \%-2.00 * 10^{3} / \mu \mathrm{l}\right)$. Se realizó una resonancia magnética de encéfalo que mostró imágenes hiperdensas en T2, FLAIR, que restringían en difusión, que no realzaban tras la administración de contraste endovenoso, distribuidas en centros semiovales y periventriculares (ver Figura 2).

\section{Figura 2. Resonancia de encéfalo, corte axial del paciente} del caso 2

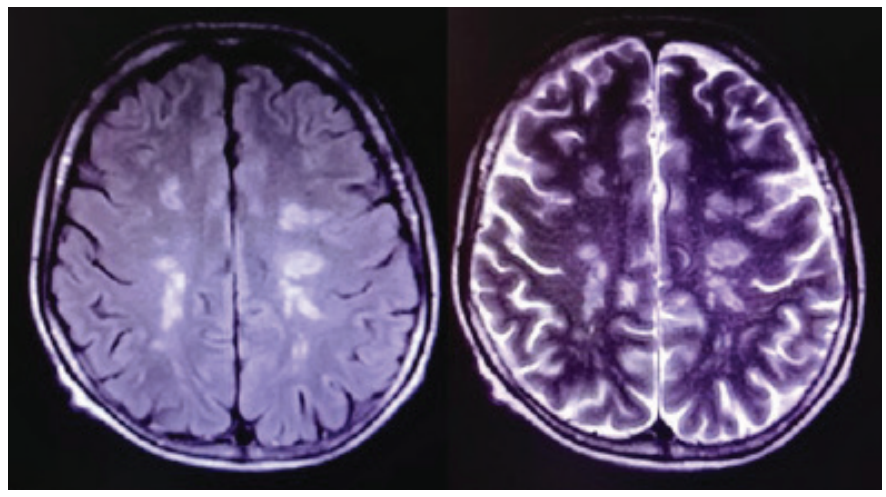

Se observan imágenes hiperdensas en T2, FLAIR, que restringen en difusión, que no realzan tras la administración de contraste EV, distribuidas en centros semiovales y periventriculares, compatibles con la patología de base.

En el contexto del brote en curso se interpretó el cuadro como triquinosis con compromiso cerebral y se internó en la sala de clínica médica, donde permaneció por catorce días, intercurriendo con episodios de confusión y excitación psicomotriz. Se realizó tratamiento con mebendazol y corticoides por diez días. Evolucionó con mejoría del sensorio, por lo que fue dado de alta. Se recibió serología IgG para Triquinella spiralis negativa al inicio y positiva a las dos semanas (título 1/128).

A los cuatro meses del alta hospitalaria se realizó una resonancia magnética de encéfalo control que informó persistencia de las imágenes anteriormente descriptas, con disminución del tamaño de las mismas y de la intensidad intralesional.
Actualmente el paciente se encuentra lúcido y relata persistencia de la debilidad generalizada en cuatro miembros, mialgias en miembros superiores, dolor cervical y paresia grado IV en miembro inferior derecho, que le impiden desarrollar sus tareas habituales.

\section{Discusión}

De acuerdo a la bibliografía, el período de incubación de la triquinosis va de 7 a 30 días y varía de acuerdo a la cantidad de larvas ingeridas. Períodos de incubación más cortos generalmente se relacionan con cursos de enfermedad más severos (6).

El compromiso cardíaco en pacientes con triquinosis fue sospechado por primera vez en 1860 por Virchow y Zenker, quienes observaron larvas del parásito en el fluido pericárdico y en el miocardio de pacientes fallecidos (2). La prevalencia de manifestaciones cardíacas varía ampliamente en la bibliografía, aunque existen pocos estudios sobre el tema. Una revisión de 17 estudios epidemiológicos de triquinosis que incluyó 5268 casos confirmó que el compromiso cardiovascular estaba presente en el $26 \%$ de los pacientes (con un rango de 1,5 a 75\%) (2). En un estudio retrospectivo que incluyó 154 casos con triquinosis, el 56\% presentó anomalías electrocardiográficas, principalmente cambios inespecíficos de la onda T-ST, seguido de alteraciones en la conducción y taquicardia sinusal. El $12 \%$ de los pacientes se identificaron como casos de miocarditis y uno presentó un infarto agudo de miocardio 28 días luego del inicio de la enfermedad y murió poco después. La autopsia reveló necrosis múltiple y fibrosis del miocardio, con un trombo en una arteria coronaria (3). En 1972 aparece el primer reporte de un caso de triquinosis que se presentó con infarto agudo de miocardio. El mismo fue en un paciente de 20 años que ingresó al hospital por dolor retroesternal intenso de varias horas de evolución. Dos días previos a la consulta había notado fiebre baja, malestar y dolor en hombros y muslos (7). Otra revisión retrospectiva que incluyó diez pacientes pertenecientes a un brote por consumo de carne de oso relata un caso que se presentó con infarto agudo de miocardio con elevación del ST. El mismo ocurrió dos semanas luego de la ingesta de la carne. El paciente consultó por fiebre, dolor de pecho con mialgias difusas, con historia de dos semanas de diarrea acuosa, fiebre y fatiga. El paciente resolvió la función cardíaca luego de la trombólisis (8).

En cuanto al compromiso neurológico, el mismo puede ocurrir 
en el 0,2 al $52 \%$ de los casos con triquinosis dependiendo de la bibliografía, apareciendo generalmente en los pacientes más severamente enfermos. La triquinosis cerebral puede afectar la sustancia blanca o gris del cerebro, el cerebelo o la médula espinal. Las larvas pueden migrar al sistema nervioso central y causar lesiones difusas, obstrucción de los vasos sanguíneos e infiltrados inflamatorios; o los productos liberados de los músculos destruidos por las larvas pueden mediar diferentes alteraciones patológicas. El compromiso neurológico de la triquinosis puede manifestarse por una variedad de signos y síntomas dependiendo de la zona afectada (9). La tomografía y resonancia de encéfalo suelen mostrar múltiples imágenes pequeñas y multifocales localizadas en la corteza cerebral y en la sustancia blanca (4). En una revisión de 77 pacientes romanos con neurotriquinosis, el $60 \%$ se recuperó completamente, el $23 \%$ quedó con secuelas y el $17 \%$ falleció. Los hallazgos patológicos en los casos fatales incluyeron edema, hemorragia, émbolos, infartos e infiltrados perivasculares (9).

La ausencia de eosinofilia en pacientes con afección del sistema nervioso o cardíaca se ha asociado en la bibliografía a peor pronóstico. En la revisión de 17 estudios de casos con afectación cardíaca, los pacientes que murieron tuvieron significativamente menos recuento de eosinóflilos que los que se recuperaron completamente (2). Un similar hallazgo fue observado en la misma revisión cuando analizaron los pacientes con compromiso neurológico (9).

Debido a su infrecuencia, existen pocos casos reportados en la bibliografía de triquinosis con compromiso cardiovascular y neurológico concomitante. La revisión que incluyó 5268 pacientes con triquinosis encontró $17(0,3 \%)$ con manifestaciones cardíacas y neurológicas simultáneamente (2). Dentro de estos pacientes se incluyen los ocho casos que publicó Fourestie et al. en 1993 (10).

La existencia de triquinosis crónica como entidad patológica sigue siendo un tema de debate. Un trabajo realizó seguimiento prospectivo por diez años de 128 pacientes con triquinosis pertenecientes a un brote. Los síntomas que se documentaron con mayor frecuencia fueron los musculares (90\%), oculares (59\%), neurológicos (52\%) y psicológicos (52\%), y dentro de estas categorías, la fuerza muscular, la conjuntivitis y la coordinación alterada fueron las manifestaciones clínicas más frecuentes (11).

En este trabajo, el paciente del primer caso presentó inicio de la sintomatología gastrointestinal a las 24 horas del consumo de los chacinados, siendo este tiempo menor al descrito en la literatura y coincidiendo con la gravedad de presentación de la enfermedad. A pesar de que las alteraciones electrocardiográficas parecen ser bastante frecuentes en los pacientes con triquinosis, el infarto agudo de miocardio con elevación del ST -como el caso que se presenta- ha sido poco reportado en la bibliografía. Este paciente presentó, además, compromiso neurológico y cardíaco concomitantes y ausencia de eosinofilia, ambos marcadores de gravedad y mal pronóstico.

Los dos pacientes con compromiso neurológico que se presentan tuvieron diferente tipo de lesión cerebral. En uno, el compromiso se manifestó como grandes áreas de isquemia, mientras en el otro se observaron múltiples imágenes multifocales en la sustancia blanca.

Por último, los dos pacientes de este trabajo persistieron con secuelas luego de presentar triquinosis y a pesar de haber realizado tratamiento específico.

El brote al que pertenecen los casos presentados, en comparación con los previos documentados en la cuidad, afectó a menos personas, pero se presentó con manifestaciones clínicas más graves e inusuales probablemente debido al consumo de chacinados con alta carga parasitaria. Es necesario conocer las posibles manifestaciones graves asociadas a la triquinosis para sospechar la patología e instaurar la terapia específica lo antes posible. 


\section{Referencias}

1. Rawla P, Sharma S. Trichinella Spiralis (Trichnellosis). Source StatPearls [Internet]. Treasure Island (FL): StatPearls Publishing; 2019. 2019 Jun 4.

2. Neghina $R$, Neghina AM, Marincu. Reviews on Trichinellosis (III): Cardiovascular Involvement. Foodborne Pathog Dis. 2011 Aug;8(8):853-60.

3. Puljizl1,BeusA,Kuzmanl,SeiwerthS.Electrocardiographic changes and myocarditis in trichinellosis: a retrospective study of 154 patients. Ann Trop Med Parasitol. 2005 Jun;99(4):403-11.

4. Gelal F, Kumral E, Vidinli BD, Erdogan D, Yucel K, Erdogan $\mathrm{N}$. Diffusion-weighted and conventional MR imaging in neurotrichinosis. Acta Radiol. 2005;46(2):196.

5. Teunis PF, Koningstein M, Takumi K, van der Giessen JW. Human beings are highly susceptible to low doses of Trichinella spp. Epidemiol Infect 2012; 140:210.

6. Kociecka W. Trichinellosis: human disease, diagnosis and treatment. Vet Parasitol 2000; 93:365.

7. Kirschberg GJ.Trichinosis presenting as acutemyocardial infarction. Can Med Assoc J 1972;106:898-899.

8. Dalcin D, Zarlenga DS, Larter NC, Hoberg E, Boucher DA, Merrifield S, et al. Trichinella Nativa Outbreak With Rare Thrombotic Complications Associated With Meat From a Black Bear. Clin Infect Dis. 2017 May 15;64(10):13671373.

9. Neghina R, Neghina AM, Marincu I, lacobiciu I. Reviews on trichinellosis (II): neurological involvement. Foodborne Pathog Dis. 2011 May;8(5):579-85.

10. Fourestie V, Douceron $\mathrm{H}$, Brugieres $\mathrm{P}$, Ancelle T, Lejonc JL, Gherardi RK. Neurotrichinosis. A cerebrovascular disease associated with myocardial injury and hypereosinophilia. Brain. 1993 Jun;116 ( Pt 3):603-16.

11. Harms $G$, Binz $P$, Feldmeier $H$, Zwingenberger $K$, Schleehauf D, Dewes W, et al. Trichinosis: a prospective controlled study of patients ten years after acute infection. Clin Infect Dis 1993;17:637-643. 
Neurological and cardiovascular involvent

in patients with trichinosis

Background: Trichinosis is a parasitic infection caused by nematodes of the genus Trichinella. Cardiac involvement is not usual but represents the most frequent cause of death by trichinosis, while neurological affectation occurs mainly in severely ill patients. A greater number of larvae ingested, the shorter the incubation time and the greater the severity of the disease. We present two patients with compromise of the central nervous system and one of them cardiovascular, both belonging to an outbreak of trichinosis in the city of Bahía Blanca.

Keywords: Trichinellosis, Trichinella spiralis, Nematode Infections. 